\title{
STRUCTURE OF ESSENTIAL SPECTRA AND DISCRETE SPECTRUM OF THE ENERGY OPERATOR OF FOUR ELECTRON SYSTEMS IN THE IMPURITY HUBBARD MODEL. THIRD TRIPLET STATE

\author{
@ S.M. Tashpulatov and R.T. Parmanova
} \\ sadullatashpulatov@yandex.com,toshpul@mail.ru,togaymurodota@gmail.com
}

\author{
УДК 517.984
}

DOI: 10.33184/mnkuomsh1t-2021-10-06.30.

Рассматривается четырехэлектронная система в примесной модели Хаббарда. Описана структура существенного спектра и дискретный спектр в этой модели в случае третьего тринлетного состояния. Доказывается, что, в одномерном случае, существенный спектр системы есть объединение не более чем шестнадщать отрезков, а дискретный спектр системы состоит из не более одинадцать собственного значение.

Ключевые слова: модель Хаббарда, примесной модель Хаббарда, четырехэлектронная система, триплетное состояние, существенный спектр, дискретный спектр.

We consider of the energy operator of four electron systems in the Impurity Hubbard model and investigated the structure of essential spectra and discrete spectrum of the system in the third triplet state. We proved, that the if $\nu=1$, then the essential spectra of the operator is consists of the union of no mote than sixteen segments, and discrete spectrum of the operator is consists of no more then eleven eigenvalues.

Keywords: Hubbard model, Impurity Hubbard model, four electron systems, triplet state, essential spectra, discrete spectrum.

The Hubbard model and impurity Hubbard model is currently one of the most extensively studied multielectron models of metals [1,2]. But little is known about exact results for the spectrum and wave functions of the crystal described by the Hubbard model, and obtaining the corresponding statements is great interest problem.

We consider the energy operator $H$ of four-electron systems in the Impurity Hubbard model, where $H=A \sum_{m, \gamma} a_{m, \gamma}^{+} a_{m, \gamma}+B \sum_{m, \tau, \gamma} a_{m, \gamma}^{+} a_{m+\tau, \gamma}+$ $U \sum_{m} a_{m, \uparrow}^{+} a_{m, \uparrow} a_{m, \downarrow}^{+} a_{m, \downarrow}+\left(A_{0}-A\right) \sum_{\gamma} a_{0, \gamma}^{+} a_{0, \gamma}+\left(B_{0}-B\right) \sum_{\tau, \gamma}\left(a_{0, \gamma}^{+} a_{\tau, \gamma}+\right.$ $\left.a_{\tau, \gamma}^{+} a_{0, \gamma}\right)+\left(U_{0}-U\right) a_{0, \uparrow}^{+} a_{0, \uparrow} a_{0, \downarrow}^{+} a_{0, \downarrow}$. Here $A\left(A_{0}\right)$ is the electron energy at

Sa'dulla Tashpulatov (Doctor of Physics and Mathematics, Leading Researcher, Institute of Nuclear Physics of Academy of Sciences of Republic of Uzbekistan, Tashkent, Uzbekistan)

Ruxsat Parmanova (trainee researcher,Institute of Nuclear Physics of Academy of Sciences of Republic of Uzbekistan, Tashkent, Uzbekistan) 
a regular (impurity) lattice site, $B\left(B_{0}\right)$ is the transfer integral between (between electron and impurities) neighboring sites (we assume that $B>$ $0\left(B_{0}>0\right)$ for convenience), $\tau= \pm e_{j}, j=1,2, \ldots, \nu$, where $e_{j}$ are unit mutually orthogonal vectors, which means that summation is taken over the nearest neighbors, $U\left(U_{0}\right)$ is the parameter of the on-site Coulomb interaction of two electrons in the regular (impurity) sites, $\gamma$ is the spin index, $\gamma=\uparrow$ or $\gamma=\downarrow$, and $a_{m, \gamma}^{+}$and $a_{m, \gamma}$ are the respective electron creation and annihilation operators at a site $m \in Z^{\nu}$.

In the four electron systems has a quintet state, and two type singlet state, and three type triplet states. The third triplet state corresponds to the free motion of four electrons over the lattice and their interactions with the basic functions: ${ }^{3} t_{p, q, r, t \in Z^{\nu}}^{1}=a_{p, \uparrow}^{+} a_{q, \downarrow}^{+} a_{r, \uparrow}^{+} a_{t, \uparrow}^{+} \varphi_{0}$. The subspace ${ }^{3} \widetilde{\mathcal{H}}_{t}^{1}$, corresponding to the third triplet state is the set of all vectors of the form ${ }^{3} \psi_{t}^{1}=\sum_{p, q, r, t \in Z^{\nu}} f(p, q, r, t)^{3} t_{p, q, r, t}^{1}, f \in l_{2}^{a s}$, where $l_{2}^{a s}$ is the subspace of antisymmetric functions in $l_{2}\left(\left(Z^{\nu}\right)^{4}\right)$. In this case, the Hamiltonian $H$ acts in the antisymmetric Foc'k space $\widetilde{\mathcal{H}}_{a s}$. Let $\varphi_{0}$ be the vacuum vector in the antisymmetrical Foc'k space $\widetilde{\mathcal{H}}_{a s}$. Let ${ }^{3} \widetilde{H}_{t}^{1}$ be the restriction $H$ to the subspace ${ }^{3} \widetilde{\mathcal{H}}_{t}^{1}$.

Theorem 1. The subspace ${ }^{3} \mathcal{H}_{1}^{t}$ is invariant under the operator $H$, and the operator ${ }^{3} H_{1}^{t}$ is a bounded self-adjoint operator. It generates a bounded self-adjoint operator ${ }^{3} \bar{H}_{1}^{t}$ acting in the space $l_{2}^{\text {as }}$.

We set ${ }^{3} \widetilde{H}_{1}^{t}=\mathcal{F}^{3} \bar{H}_{1}^{t} \mathcal{F}^{-1}$. In the quasimomentum representation, the operator ${ }^{3} \widetilde{H}_{1}^{t}$ acts in the Hilbert space $L_{2}^{a s}\left(\left(T^{\nu}\right)^{4}\right)$.

Theorem 2. Let $\nu=1$, and $\varepsilon_{2}=-B$, and $\varepsilon_{1}<-2 B$ (respectively, $\varepsilon_{2}=-B$, and $\varepsilon_{1}>2 B$. Then the essential spectrum of the operator ${ }^{3} \widetilde{H}_{1}^{t}$ is consists of the union of $N_{1}$ segments, where $4 \leq N_{1} \leq 8: \sigma_{\text {ess }}\left({ }^{3} \widetilde{H}_{1}^{t}\right)=$ $[4 A-8 B, 4 A+8 B] \cup[3 A-6 B+z, 3 A+6 B+z] \cup[2 A-4 B+2 z, 2 A+4 B+2 z] \cup$ $[A-2 B+3 z, A+2 B+3 z] \cup\left[2 A-4 B+z_{3}, 2 A+4 B+z_{3}\right] \cup\left[2 A-4 B+z_{4}, 2 A+4 B+\right.$ $\left.z_{4}\right] \cup\left[A-2 B+z+z_{3}, A+2 B+z+z_{3}\right] \cup\left[A-2 B+z+z_{4}, A+2 B+z+z_{4}\right]$, and the discrete spectrum of operator ${ }^{3} \widetilde{H}_{1}^{t}$ is consists of no more three eigenvalues: $\sigma_{\text {disc }}\left({ }^{3} \widetilde{H}_{1}^{t}\right)=\left\{4 z, 2 z+z_{3}, 2 z+z_{4}\right\}$, where $z=A+\varepsilon_{1}$, and $z_{3}$ and $z_{4}$ are the additional eigenvalues of operator ${ }^{3} \widetilde{H}_{1}^{t}$.

Theorem 3. If $\nu=1$, and $-2 B<\varepsilon_{2}<0$, then the essential spectrum of the operator ${ }^{3} \widetilde{H}_{1}^{t}$ is consists of the union of $N_{1}$ segments, where $1 \leq N_{1} \leq 3$ : $\sigma_{\text {ess }}\left({ }^{3} \widetilde{H}_{1}^{t}\right)=[4 A-8 B, 4 A+8 B] \cup\left[2 A-4 B+z_{3}, 2 A+4 B+z_{3}\right] \cup[2 A-4 B+$ $\left.z_{4}, 2 A+4 B+z_{4}\right]$, and the discrete spectrum of operator ${ }^{3} \widetilde{H}_{1}^{t}$ is empty set.

Theorem 4. Let $\nu=1$, and $\varepsilon_{2}>0$, and $-\frac{2\left(\varepsilon_{2}^{2}+2 B \varepsilon_{2}\right)}{B}<\varepsilon_{1}<\frac{2\left(\varepsilon_{2}^{2}+2 B \varepsilon_{2}\right)}{B}$, then the essential spectrum of the operator ${ }^{3} \widetilde{H}_{1}^{t}$ is consists of the union of the $N_{1}$ segment, where $10 \leq N_{1} \leq 16: \sigma_{\text {ess }}\left({ }^{3} \widetilde{H}_{1}^{t}\right)=[4 A-8 B, 4 A+8 B] \cup$ 
$\left[3 A-6 B+z_{1}, 3 A-6 B+z_{1}\right] \cup\left[3 A-6 B+z_{2}, 3 A-6 B+z_{2}\right] \cup[2 A-4 B+$ $\left.2 z_{1}, 2 A+4 B+2 z_{1}\right] \cup\left[2 A-4 B+2 z_{2}, 2 A+4 B+2 z_{2}\right] \cup\left[2 A-4 B+z_{1}+z_{2}, 2 A+\right.$ $\left.4 B+z_{1}+z_{2}\right] \cup\left[A-2 B+3 z_{1}, A+2 B+3 z_{1}\right] \cup\left[A-2 B+3 z_{2}, A+2 B+3 z_{2}\right] \cup$ $\left[A-2 B+2 z_{1}+z_{2}, A+2 B+2 z_{1}+z_{2}\right] \cup\left[A-2 B+z_{1}+2 z_{2}, A+2 B+z_{1}+\right.$ $\left.2 z_{2}\right] \cup\left[2 A-4 B+z_{3}, 2 A+4 B+z_{3}\right] \cup\left[A-2 B+z_{1}+z_{3}, A+2 B+z_{1}+z_{3}\right] \cup$ $\left[A-2 B+z_{2}+z_{3}, A+2 B+z_{2}+z_{3}\right] \cup\left[2 A-4 B+z_{4}, 2 A+4 B+z_{4}\right] \cup[A-$ $\left.2 B+z_{1}+z_{4}, A+2 B+z_{1}+z_{4}\right] \cup\left[A-2 B+z_{2}+z_{4}, A+2 B+z_{2}+z_{4}\right]$ and discrete spectrum of the operator ${ }^{3} \widetilde{H}_{1}^{t}$ is consists of $N_{2}$ eigenvalues, where $5 \leq N_{2} \leq 11: \sigma_{d i s c}\left(\widetilde{H}_{1}^{t}\right)=\left\{4 z_{1}, 4 z_{2}, 3 z_{1}+z_{2}, z_{1}+3 z_{2}, 2 z_{1}+2 z_{2}, 2 z_{1}+\right.$ $\left.z_{3}, z_{1}+z_{2}+z_{3}, 2 z_{2}+z_{3}, 2 z_{1}+z_{4}, z_{1}+z_{2}+z_{4}, 2 z_{2}+z_{4}\right\}$.

\section{Литература}

1. Hubbard J. Electron Correlations in Narrow Energy Band // Proc. Roy. Soc. A. 276:1365 (1963), 238- 257.

2. Karpenko B.V., Dyakin V.V., and Budrina G.L. Two electrons in the Hubbard Model.// Phys. Met. Metallogr. 61 (1986), 702-706. 